\title{
Towards a physical interpretation for the Stephani Universes.
}

\author{
Roberto A. Sussman \\ Facultad de Ciencias, UAEM, Av. Universidad 1001, Cuernavaca, Morelos, 62145, México.*
}

(March 23, 2018)

\begin{abstract}
A physicaly reasonable interpretation is provided for the perfect fluid, sphericaly symmetric, conformally flat "Stephani Universes". The free parameters of this class of exact solutions are determined so that the ideal gas relation $p=n k_{B} T$ is identicaly fulfiled, while the full equation of state of a classical monatomic ideal gas and a matter-radiation mixture holds up to a good approximation in a near dust, matter dominated regime. Only the models having spacelike slices with positive curvature admit a regular evolution domain that avoids an unphysical singularity. In the matter dominated regime these models are dynamicaly and observationaly indistinguishable from "standard" FLRW cosmology with a dust source.
\end{abstract}

\section{INTRODUCTION.}

The most general class of non-static, perfect fluid solutions of Einstein's equations that are conformally flat is known as the "Stephani Universe" [1], [2]. These mathematicaly simple solutions are characterized (in general) by a shear-free but expanding and accelerating 4-velocity, with perfect fluid FLRW spacetimes being the acceleration free subcase. The sphericaly symmetric Stephani Universes 1 and some of their subcases have been examined in numerous papers (see [2] for a comprehensive review). For example, as star models [3], [4], [5], [6], [7] and as cosmologies generalizing FLRW [2], 8], [9]. Other papers have looked at their global properties and singularities [10], [11], [12], [13], [14], [15], the thermodynamics of their fluid source [16], 17], [18], and, more recently, as models with inhomogeneous pressure in which to test recent astronomical data related to supernova observations [19], [20]. Although these solutions are popular because of their mathematical simplicity, they are not considered physicaly meaningful because their fluid source seems quite artificial and excessively restrictive: matter-energy density, $\rho$, depends only on $t$ while pressure, $p$, depends on $t$ and $r$ (in comoving coordinates). Another objection to the Stephani Universes is their incompatibility with a barotropic relation [21], 22] of the form $p=p(\rho)$. However, the lack of fulfilment of barotropic relations is not a strong argument to dismiss a given fluid solution, since the latter might be compatible with more meaningful non-barotropic equations of state.

The thermodynamics of the fluid source of the Stephani Universes is given by the balance equations of energy, momentum and entropy per particle, as well as by the particle number conservation and the equilibrium Gibbs equation. Previous papers have shown that the general Stephani Universe, having as source a single component perfect fluid, is incompatible with the integrability conditions of the Gibbs equation [16], [18], so that a " thermodynamical scheme" is not possible (or in other words, a mathematicaly consistent and closed set of thermodynamical equations of state is incompatible with the field equations). However, this restriction does not apply to the sphericaly symmetric case, or to any subcase whose isometry group has orbits of dimension 2 or larger, hence mathematicaly simple equations of state have been found [17] satisfying energy conditions, but without any basis on actual physical matter models 2 .

Accepting the above mentioned limitations, this paper aims at looking for a reasonable (or at least, less objectionable) interpretation for the Stephani Universes. Using the Gibbs equation as a definition of temperature, $T$, it is posible to determine the four free functions by imposing the constraint: $p=n k_{B} T$, where $n$ is particle number density and $k_{B}$ is Boltzmann's constant. However, the fulfilment of the relation between $\rho$ and $p$ characteristic of the equation of state of the ideal gas [23] (together with $p=n k_{B} T$ ) cannot hold because $\rho=\rho(t)$ while $p=p(t, r)$. We consider the equations of state of: (A) a single component classical monatomic ideal gas, and (B) a mixture of non-relativistic and relativistic ideal gases, where the internal energy of the former has been ignored (hence, $p$ and $T$ are the radiation pressure and temperature). By writing up the explicit form of the state variables for the forms of the free functions that satisfy $p=n k_{B} T$, we provide explicitly the conditions for a tight approximation to the full

\footnotetext{
* On sabatical leave from Instituto de Ciencias Nucleares, Apartado Postal 70543, UNAM, México DF, 04510, México. Email sussman@nuclecu.unam.mx.

${ }^{1}$ Unless specificaly stated otherwise, all further mention of "Stephani Universes" will refere to sphericaly symmetric Stephani Univeses excluding their FLRW particular subclass

${ }^{2}$ The subclass of Stephani Universes studied in ref [17] was erroneously reported as lacking isometries when it is sphericaly symmetric. See [2] and [18]
} 
equations of state (A) and (B) in terms of the convergence of a series of powers of adimensional products of $\rho$ and a parameter $\varepsilon_{0}$, proportional to the energy ratio $k_{B} \hat{T}_{0} / m c^{2}$, where $\hat{T}_{0}$ is a suitable constant temperature value and $m$ is the particles mass. This series converges in a matter dominated low temperature regime characterized by $\varepsilon_{0} \ll 1$, for models having spacelike slices (hypersurfaces of constant $t$, orthogonal to the 4 -velocity) with constant positive curvature (like "closed" FLRW cosmologies). Under this approximation, the evolution of the fluid in the matter dominated regime becomes very close to a dust FLRW cosmology, to such a degree that observational parameters $H_{0}, \Omega_{0}, q_{0}$ become practicaly indistinguishable from those of the FLRW spacetime. The same models for which the above mentioned series converges are the only ones admiting an evolution range that avoids unphysical features, such as a "Finite Density" singularity and an "Asymptoticaly deSitter boundary" (see [12] to [15]), and so allowing for a regular initial Cauchy hypersurface. Models whose spacelike slices orthogonal to the 4-velocity have zero or negative curvature lack a regular Cauchy hypersurface, while the series governing the approximation to the ideal gas equations of state (A) and (B) only converge for comoving observers near the symmetry center $r=0$.

Considering their qualities and defects, the subclass of Stephani Universes derived and discussed in the present paper provide a reasonable and theoreticaly consistent interpretation for this simple and well established class of exact solutions.

\section{THE SPHERICALY SYMMETRIC STEPHANI UNIVERSES.}

The metric in comoving coordinates for the sphericaly symmetric Stephani Universes is usualy given in such a way as to emphasize its resemblance with FLRW cosmologies [2]. However, we shall use instead the following alternative comoving coordinates:

$$
\begin{gathered}
d s^{2}=-U^{2} c^{2} d t^{2}+L^{2}\left[d r^{2}+f^{2}\left(d \theta^{2}+\sin ^{2} \theta d \varphi^{2}\right)\right] \\
U=\frac{1+\left(K-R K_{, R}\right) F^{2}}{1+K F^{2}}, \quad L=\frac{R}{1+K F^{2}}
\end{gathered}
$$

where $R=R(t), K=K(t)$ and $K_{, R}=K_{, t} / R_{, t}=d K / d R$, and the functions $F(r), f(r)$ are given by the three possible combinations

$$
\begin{aligned}
f=r, & F=r / 2 \\
f=\sin r, & F=\sin (r / 2) \\
f=\sinh r, & F=\sinh (r / 2)
\end{aligned}
$$

The transformation of the radial coordinate $r$, relating (1)-(2) with the usual radial coordinate for the Stephani Universes, $\bar{r}$, is given by $r=\int d \bar{r} /\left(1+k \bar{r}^{2} / 4\right)$, where $k=0, \pm 1$. The matter tensor associated with (1) is a perfect fluid

$$
\begin{gathered}
T^{a b}=\rho u^{a} u^{b}+p h^{a b} \\
u^{a}=\frac{1}{U} \delta_{t}^{a}
\end{gathered}
$$

where $h^{a b}=c^{-2} u^{a} u^{b}+g^{a b}$ and $\rho, p$ are matter energy density and equilibrium pressure. The time coordinate in (1) has been selected so that the expansion scalar $\Theta=u_{; a}^{a}$ is given by

$$
\frac{\Theta}{3}=\frac{R_{, t}}{R}
$$

The fluid source of the Stephani Universes is shear-free, hence the remaining nonzero kinematic scalar is the 4acceleration: $\dot{u}_{a} \equiv u_{a ; b} u^{b}$, whose explicit form for (1) is

$$
\dot{u}_{a}=\frac{U_{, r}}{U} \delta_{a}^{r}=-\frac{R K_{, R} f}{2\left[1+K F^{2}\right]\left[1+\left(K-R K_{, R}\right) F^{2}\right]} \delta_{a}^{r}
$$


A perfect fluid source like (2) satisfies energy-momentum balance: $T_{; b}^{a b}=0$, particle number conservation $\left(n u^{a}\right)_{; a}=$ 0 and entropy balance: $\left(n s u^{a}\right)_{; a}=0$, where $n, s$ are particle number density and entropy per particle. These balance and conservation laws take the following forms for (1) and (2)

$$
\begin{gathered}
u_{a} T_{; b}^{a b}=\dot{\rho}+(\rho+p) \Theta=0 \\
h_{a b} T_{; c}^{b c}=h_{a}{ }^{b} p_{, b}+(\rho+p) \dot{u}_{a}=0 \\
\left(n u^{a}\right)_{; a}=\dot{n}+n \Theta=0 \\
\left(n s u^{a}\right)_{; a}=(\dot{n}+n \Theta) s+n \dot{s}=0 \Rightarrow \dot{s}=0
\end{gathered}
$$

where $\dot{A}=u^{a} A_{a}=u^{t} A_{, t}$ is the proper time derivative for any scalar function $A$ and $\Theta, \dot{u}_{a}$ are given by (5) and (6). The state variables must also comply with the equilibrium Gibbs equation

$$
T \mathrm{ds}=\mathrm{d}\left(\frac{\rho}{\mathrm{n}}\right)+\operatorname{pd}\left(\frac{1}{\mathrm{n}}\right)
$$

where $T$ is absolute temperature.

The field equation $G^{t}{ }_{t}=\left(8 \pi G / 3 c^{2}\right) T^{t}{ }_{t}$ for (1), (2) and (3) is

$$
\frac{8 \pi G}{3 c^{2}} \rho=\left(\frac{R_{, t}}{R}\right)^{2}+\frac{(K+k) c^{2}}{R^{2}}
$$

where:

$$
k=\left\{\begin{array}{cl}
0, & f=r, \quad F=r / 2, \\
1, & f=\sin (r), \quad F=\sin (r / 2), \\
-1, & f=\sinh (r), \quad F=\sinh (r / 2),
\end{array}\right.
$$

while the form of $p$ follows from (7a) as

$$
p=-\rho-\frac{R}{3} \frac{\rho, R}{U}=-\rho-\frac{R}{3} \rho_{, R}\left[\frac{1+K F^{2}}{1+\left(K-R K_{, R}\right) F^{2}}\right]
$$

The momentum ballance $(7 \mathrm{~b})$ is

$$
p^{\prime}+\frac{f R^{2} \rho_{, R} K_{, R}}{6\left[1+\left(K-R K_{, R}\right) F^{2}\right]^{2}}=0
$$

where a prime denotes derivative wrt to $r$ and (6) and (11) were used to eliminate $\dot{u}_{a}$ and $\rho+p$. The particle number density is given by integrating $(7 \mathrm{c})$

$$
n=\frac{N(r)}{R^{3}}\left[1+K F^{2}\right]^{3}
$$

where $N$ is the conserved particle number distribution. Since the spacial coordinates in (1) are comoving, the entropy balance (7d) yields simply $s=s(r)$, and so, with the help of (7a) and (7c), the Gibbs equation (8) reduces to

$$
T s^{\prime}=(\rho+p)\left(\frac{1}{n}\right)^{\prime}
$$

with $s^{\prime} \neq 0$, since the fluid of Stephani Universes is not isentropic (or equivalently, does not admit a barotropic relation $p=p(\rho))$. 


\section{DETERMINATION OF THE FREE PARAMETERS.}

The Stephani Universes contain (in general) two time dependent free functions $(R(t), K(t)$ in $(1))$ whose determination is elusive. The imposition of an equation of state (ie a constraint relating state variables) should lead to the determination of these free-parameters, howewer not all relations between the state variables is compatible with these free functions and with the radial dependence of the state variables (in particular, the barotropic relation $p=p(\rho)$ is incompatible, see [21], 222]). Conversely, if the two free functions are determined by suitable boundary conditions, or prescribed in order to obtain specific kinematic effects, a specific constraint among state variables ensues, though such constraint is a "formal" equation of state and might be wholy unphysical. The usual boundary conditions invoked for determining the free functions are: a matching with a Schwarzschild vacuum exterior along a comoving boundary

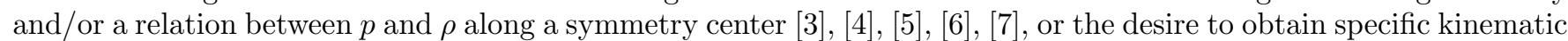
efects, like bouncing [3] or acceleration of comoving observers [19], [20], or a metric element that is conformal to that of a FLRW metric [8], [9], 14], 19], [20]. Besides the time dependent free parameters, a proper thermodynamical approach produces two extra free functions of $r$ : the initial particle number distribution, $N(r)$, and the entropy per particle $s(r)$.

The Stephani Universes, as exact solutions subjected to the thermodynamical laws (7c), (7d) and (8), become determined once the four free functions $R(t), K(t), N(r), s(r)$ are either prescribed, found or obtained. From equations (1)-(14), once $\rho$ and $K$ are known as functions of $R$, the field equation (9) becomes the Friedmann equation whose integration yields $R$ as a function of $t$. However, the functions $N(r), s(r)$ cannot be determined from the geometry but from thermodynamical criteria. The usual strategies to determine the free functions have lead, in all cases, to equations of state whose physics has still remained unclear. We propose in this paper another strategy for the determination of these free functions.

Consider the following relation associated with the equation of state of an ideal gas:

$$
p=n k_{B} T
$$

where $k_{B}$ is Boltzmann's constant. Using $p, n$ from (11) and (13), the fulfilment of (15) implies the constraint

$$
k_{B} T=\frac{k_{B}(\rho+p)}{s^{\prime}}\left(\frac{1}{n}\right)^{\prime}=\frac{p}{n}
$$

which becomes explicitly

$$
\left(1+K F^{2}\right)\left[\rho+\frac{R \rho_{, R}}{3}\left(1+\frac{k_{B} N^{\prime}}{s^{\prime} N}\right)\right]+R F\left[\frac{2 k_{B} F^{\prime}}{s^{\prime}} K \rho_{, R}-\rho K_{, R} F\right]=0
$$

The only physicaly reasonable solution of (16) (other solutions yield negative values of $n$ and $T$ ) follows if the terms in square parenthesis vanish independently. Since $\rho, K$ depend on $R$ while $F, N, s$ depend on $r$, we have

$$
\begin{gathered}
\frac{R \rho_{, R}}{3 \rho}=-\left(1+\frac{k_{B} N^{\prime}}{s^{\prime} N}\right)^{-1}=a \\
\frac{2 k_{B} F^{\prime}}{s^{\prime} F}=\frac{K_{, R} / K}{\rho_{, R} / \rho}=b
\end{gathered}
$$

where $a, b$ are arbitrary nonzero real constants. Equations (17) lead to

$$
\begin{gathered}
\rho=\rho_{0}\left(\frac{R_{0}}{R}\right)^{3 a}, \quad K=k_{1}\left(\frac{\rho}{\rho_{0}}\right)^{b}=k_{1}\left(\frac{R_{0}}{R}\right)^{3 a b} \\
\log N=\frac{2(1-a)}{b} \log F, \quad s=s_{0}+\frac{a k_{B}}{1-a} \log N, \quad a \neq 1 \\
N=N_{0}, \quad s=s_{0}+\frac{2 k_{B}}{b} \log F, \quad a=1
\end{gathered}
$$


Inserting (18) into (9), (11), (13) and using (15) leads to

$$
\begin{gathered}
\left(\frac{R_{, t}}{R}\right)^{2}=\frac{8 \pi G}{c^{2}} \rho_{0}\left(\frac{R_{0}}{R}\right)^{3 a}-\frac{k_{1} c^{2}}{R^{2}}\left(\frac{R_{0}}{R}\right)^{3 a b}-\frac{k_{0} c^{2}}{R^{2}} \\
p=\frac{(a-1) \rho_{0}+[(1-3 b) a-1] \rho_{0} k_{1}\left(R_{0} / R\right)^{3 a b} F^{2}}{1+(1+3 a b) k_{1}\left(R_{0} / R\right)^{3 a b} F^{2}}\left(\frac{R_{0}}{R}\right)^{3 a} \\
n=\frac{N}{R^{3}}\left[1+k_{1}\left(\frac{R_{0}}{R}\right)^{3 a b} F^{2}\right]^{3} \\
k_{B} T=\frac{R_{0}^{3}}{N} \frac{(a-1) \rho_{0}+[(1-3 b) a-1] \rho_{0} k_{1}\left(R_{0} / R\right)^{3 a b} F^{2}}{\left[1+k_{1}\left(R_{0} / R\right)^{3 a b} F^{2}\right]^{3}\left[1+(1+3 a b) k_{1}\left(R_{0} / R\right)^{3 a b} F^{2}\right]}\left(\frac{R_{0}}{R}\right)^{3(a-1)}
\end{gathered}
$$

From (18d), (18d), (19c) and (19d) it is evident that the choice $a \neq 1$ leads to a diverging temperature along a symmetry center marked by $F(0)=0$. Hence, we must consider only $a=1, b>0$, but in this case, in order to have positive $p$ and $T$, we must also assume $k_{1}<0$. It turns to be convenient to redefine the constant parameters $b$ and $k_{1}$ as

$$
b=\gamma-1, \quad-k_{1}=\frac{\varepsilon_{0}}{3(\gamma-1)}
$$

where $\varepsilon_{0}$ is a positive constant. The metric functions in (1), 4-velocity and 4-acceleration (4) and (6) become

$$
\begin{gathered}
U=\frac{1-\varepsilon_{0}\left(\gamma_{2} / \gamma_{1}\right) F^{2}\left(R_{0} / R\right)^{\gamma_{1}}}{1-\left(\varepsilon_{0} / \gamma_{1}\right) F^{2}\left(R_{0} / R\right)^{\gamma_{1}}}, \quad L=\frac{R}{1-\left(\varepsilon_{0} / \gamma_{1}\right) F^{2}\left(R_{0} / R\right)^{\gamma_{1}}} \\
u^{a}=\frac{1}{U} \delta_{t}^{a}, \quad \dot{u}_{a}=\aleph \delta_{a}^{r}, \quad \aleph=\frac{\frac{1}{2} \varepsilon_{0}\left(R / R_{0}\right)^{\gamma_{1}} f}{\left[\left(R / R_{0}\right)^{\gamma_{1}}-\left(\varepsilon_{0} / \gamma_{1}\right) F^{2}\right]\left[\left(R / R_{0}\right)^{\gamma_{1}}-\varepsilon_{0}\left(\gamma_{2} / \gamma_{1}\right) F^{2}\right]}
\end{gathered}
$$

where

$$
\gamma_{1}=3(\gamma-1), \quad \gamma_{2}=3 \gamma-2
$$

The state variables in (19) take the forms

$$
\begin{gathered}
\rho=\rho_{0}\left(\frac{R_{0}}{R}\right)^{3} \\
p=\frac{\rho_{0} \varepsilon_{0} F^{2}}{1-\varepsilon_{0}\left(\gamma_{2} / \gamma_{1}\right)\left(\rho / \rho_{0}\right)^{\gamma-1} F^{2}}\left(\frac{\rho}{\rho_{0}}\right)^{\gamma} \\
n=\frac{N_{0}}{R_{0}^{3}}\left(\frac{\rho}{\rho_{0}}\right)\left[1-\frac{\varepsilon_{0}}{\gamma_{1}}\left(\frac{\rho}{\rho_{0}}\right)^{\gamma-1} F^{2}\right]^{3} \\
k_{B} T=\frac{\rho_{0} \varepsilon_{0} R_{0}^{3} F^{2}}{N_{0}\left[1-\left(\varepsilon_{0} / \gamma_{1}\right)\left(\rho / \rho_{0}\right)^{\gamma-1} F^{2}\right]^{3}\left[1-\varepsilon_{0}\left(\gamma_{2} / \gamma_{1}\right)\left(\rho / \rho_{0}\right)^{\gamma-1} F^{2}\right]}\left(\frac{\rho}{\rho_{0}}\right)^{\gamma-1}
\end{gathered}
$$

while the Friedmann equation (9) is given by

$$
\left(R_{, t}\right)^{2}=\frac{8 \pi G \rho_{0} R_{0}^{3}}{3 c^{2} R}+\frac{\varepsilon_{0} c^{2}}{\gamma_{1}}\left(\frac{R_{0}}{R}\right)^{\gamma_{1}}-k c^{2}
$$


This equation must be integrated in order to obtain $R=R(t)$ and have the models fuly determined.

So far, we have determined the four free parameters of the Stephani Universe by imposing the constraint (15). From $(18 \mathrm{c})$ and $(22 \mathrm{a})$, the state variables in $(22 \mathrm{~b}-\mathrm{d})$ can be all be expressed as functions of two primary thermodynamical variables $(\rho, s)$, so that rewriting (8) in terms of the latter and using (15), it is straightforward to prove that the integrability conditions of this Gibbs equation are fuly satisfied. However, the equations of state of ideal gases impose, besides (15), further relations among the remaining state variables [23]. We look at two of these equations of state in the following section.

\section{EQUATIONS OF STATE.}

The fulfilment of the relation $p=n k_{B} T$ leads to the consideration of various possible ideal gas equations of state for the fluid. However, we have: $\rho=\rho(t)$, while the remaining state variables depend on both $t$ and $r$, hence these equations of state will not hold exactly, though the constant parameters $\gamma, \varepsilon_{0}, \rho_{0}$ can be determined in such a way that the full equation of state holds under suitable approximations. Two cases will be examined separately below.

\section{A. The classical monatomic ideal gas}

For this case the equation of state is (15) together with [23], 25]

$$
\rho=m c^{2} n+\frac{3}{2} n k_{B} T
$$

As mentioned before, an equation like (24) cannot be satisfied exactly. In order to examine under which conditions it can be satisfied approximately, we use (15), (22b) and (22c) to write the right hand side of (24) expanded up to third order in $\varepsilon_{0}$

$$
\begin{aligned}
m c^{2} n+\frac{3}{2} n k_{B} T= & \frac{m c^{2} N_{0}}{R_{0}^{3}}\left(\frac{\rho}{\rho_{0}}\right)+\left[\frac{3}{2} \rho_{0}-\frac{m c^{2} N_{0}}{(\gamma-1) R_{0}^{3}}\right]\left(\frac{\rho}{\rho_{0}}\right)^{\gamma} F^{2} \varepsilon_{0}+\left[\frac{3 \gamma-2}{2(\gamma-1)} \rho_{0}+\frac{m c^{2} N_{0}}{3(\gamma-1)^{2} R_{0}^{3}}\right]\left(\frac{\rho}{\rho_{0}}\right)^{2 \gamma-1} F^{4} \varepsilon_{0}^{2} \\
& +\left[\frac{(3 \gamma-2)^{2}}{6(\gamma-1)^{2}} \rho_{0}-\frac{m c^{2} N_{0}}{27(\gamma-1)^{3} R_{0}^{3}}\right]\left(\frac{\rho}{\rho_{0}}\right)^{3 \gamma-2} F^{6} \varepsilon_{0}^{3}+O\left(\varepsilon_{0}^{4}\right)
\end{aligned}
$$

Since $\rho_{0}, R_{0}$ are $\rho, R$ evaluated at present cosmic time $t=t_{0}$ when matter dominated conditions are assumed to prevail, then energy density is overwhelmingly dominated by rest mass energy, and so, looking for the best possible approximation of (25) to (24), leads to the following identifications in (25)

$$
\rho_{0}=\frac{m c^{2} N_{0}}{R_{0}{ }^{3}}, \quad \frac{1}{\gamma-1}=\frac{3}{2} \quad \Rightarrow \quad \gamma=\frac{5}{3}
$$

Inserting (26) into (25) yields

$$
m c^{2} n+\frac{3}{2} n k_{B} T=\rho\left[1+3\left(\frac{\rho}{\rho_{0}}\right)^{4 / 3} F^{4} \varepsilon_{0}^{2}+\frac{13}{4}\left(\frac{\rho}{\rho_{0}}\right)^{2} F^{6} \varepsilon_{0}^{3}+O\left(\varepsilon_{0}^{4}\right)\right]
$$

so that the leading nonzero order term in the series inside the square brackets is quadratic in $\varepsilon_{0}$. Comparing (27) with (24) shows that (22) and (26) lead to a good approximation to (24) provided the power series inside the square brackets in (27) converges. A sufficient condition for this convergence requires that each term (with the exception of the zero order term) must satisfy

$$
\left(\frac{\rho}{\rho_{0}}\right)^{2 \xi / 3} F^{2 \xi}\left(\varepsilon_{0}\right)^{\xi} \ll 1, \quad \xi=2,3, . .
$$

The form of the adimensional parameter $\varepsilon_{0}$ follows by using (22b), (22d) and (26) in order to expand $p$ and $k_{B} T$ around $\varepsilon_{0}$ 


$$
\begin{aligned}
& p=\frac{m c^{2} N_{0} \varepsilon_{0} F^{2}}{R_{0}^{3}}\left(\frac{\rho}{\rho_{0}}\right)^{5 / 3}\left[1+\frac{3}{2}\left(\frac{\rho}{\rho_{0}}\right)^{2 / 3} F^{2} \varepsilon_{0}+\frac{9}{4}\left(\frac{\rho}{\rho_{0}}\right)^{4 / 3} F^{2} \varepsilon_{0}^{2}+O\left(\varepsilon_{0}^{3}\right)\right] \\
& k_{B} T=m c^{2} \varepsilon_{0} F^{2}\left(\frac{\rho}{\rho_{0}}\right)^{2 / 3}\left[1+3\left(\frac{\rho}{\rho_{0}}\right)^{2 / 3} F^{2} \varepsilon_{0}+6\left(\frac{\rho}{\rho_{0}}\right)^{4 / 3} F^{2} \varepsilon_{0}^{2}+O\left(\varepsilon_{0}^{3}\right)\right]
\end{aligned}
$$

By looking at the common factor outside the square brackets, it becomes natural to identify

$$
m c^{2} \varepsilon_{0}=k_{B} \hat{T}_{0} \quad \Rightarrow \quad \varepsilon_{0}=\frac{k_{B} \hat{T}_{0}}{m c^{2}}
$$

where $\hat{T}_{0}$ is a suitable constant temperature value. With the help of (22), (26) and (30), the exact form of the state variables becomes

$$
\begin{gathered}
p=\frac{\left(N_{0} k_{B} \hat{T}_{0} / R_{0}^{3}\right) F^{2}}{1-\frac{3}{2} \varepsilon_{0}\left(\rho / \rho_{0}\right)^{2 / 3} F^{2}}\left(\frac{\rho}{\rho_{0}}\right)^{5 / 3}=\frac{\left(N_{0} k_{B} \hat{T}_{0} / R_{0}^{3}\right) F^{2}}{1-\frac{3}{2} \varepsilon_{0}\left(R_{0} / R\right)^{2} F^{2}}\left(\frac{R_{0}}{R}\right)^{5} \\
n=\frac{N_{0}}{R_{0}^{3}}\left(\frac{\rho}{\rho_{0}}\right)\left[1-\frac{\varepsilon_{0}}{2}\left(\frac{\rho}{\rho_{0}}\right)^{2 / 3} F^{2}\right]^{3}=\frac{N_{0}\left[1-\frac{\varepsilon_{0}}{R^{3}}\left(\frac{R_{0}}{R}\right)^{2} F^{2}\right]^{3}}{k_{B} T=\frac{k_{B} \hat{T}_{0} F^{2}}{\left[1-\frac{\varepsilon_{0}}{2}\left(\rho / \rho_{0}\right)^{2 / 3} F^{2}\right]^{3}\left[1-\frac{3}{2} \varepsilon_{0}\left(\rho / \rho_{0}\right)^{2 / 3} F^{2}\right]}\left(\frac{\rho}{\rho_{0}}\right)^{2 / 3}=\frac{k_{B} \hat{T}_{0} F^{2}}{\left[1-\frac{\varepsilon_{0}}{2}\left(R_{0} / R\right)^{2} F^{2}\right]^{3}\left[1-\frac{3}{2} \varepsilon_{0}\left(R_{0} / R\right)^{2} F^{2}\right]}\left(\frac{R_{0}}{R}\right)^{2}}
\end{gathered}
$$

while the Friedmann equation (22d) is given by

$$
\left(R_{, t}\right)^{2}=\frac{8 \pi G m N_{0}}{3 R}+\frac{\varepsilon_{0} c^{2}}{2}\left(\frac{R_{0}}{R}\right)^{2}-k c^{2}
$$

The degree in which these state variables provide a good approximation to the equation of state (15) (24) strongly depends on the behavior of $F, \rho / \rho_{0}$ and on the value of $\varepsilon_{0}$ in (30), a small number for an enormous range of temperatures (if $m$ is a protonic mass, we have $\varepsilon_{0} \approx 10^{-4}$ for as high as $\hat{T}_{0} \approx 10^{8}$ degrees K.). The fulfilment of (28) for specific energy and temperature ranges of the models will be discussed in section VII.

\section{B. Mixture of matter and radiation}

Consider a mixture of two ideal gases, one non-relativistic (superindex "I") and the other ultra-relativistic (superindex "II"), the equation of state in this case is

$$
\begin{gathered}
\rho=m c^{2} n^{\mathrm{I}}+\frac{3}{2} n^{\mathrm{I}} k_{B} T^{\mathrm{I}}+3 n^{\mathrm{II}} k_{B} T^{\mathrm{II}} \\
p=n^{\mathrm{I}} k_{B} T^{\mathrm{I}}+n^{\mathrm{II}} k_{B} T^{\mathrm{II}}
\end{gathered}
$$

If the internal energy of the non-relativistic gas can be neglected: $n^{\mathrm{I}} k_{B} T^{\mathrm{I}} \ll n^{\mathrm{II}} k_{B} T^{\mathrm{II}}$ but (at least at a given stage of the evolution) the energy density $n^{\mathrm{II}} k_{B} T^{\mathrm{II}}$ is not negligible in comparison with the rest mass energy $m c^{2} n^{\mathrm{I}}$, then (33) can be approximated by [25], 26]

$$
\begin{gathered}
\rho=m c^{2} n^{\mathrm{I}}+3 n^{\mathrm{II}} k_{B} T \\
p=n^{\mathrm{II}} k_{B} T
\end{gathered}
$$


where $T=T^{\mathrm{II}}$ and the particle number densities independently satisfy conservation laws like (7c), therefore $n^{\mathrm{I}}, n^{\mathrm{II}}$ are given by expressions identical to $(22 \mathrm{c})$ with $N_{0}$ replaced by $N_{0}^{\mathrm{I}}$ and $N_{0}^{\mathrm{II}}$. The approximation $(34 \mathrm{a}-\mathrm{b})$ can be especialy suited for a mixture of photons and barions [26] bacause the ratio of the former to the latter is such a large number $\left(\approx 10^{8}\right)$, leading to a reasonable description of cosmological mixtures of non-relativistic matter and radiation for energy and temperature ranges prevailing after cosmological nucleosynthesis, including the radiative era (recombination and decoupling), and up to the present.

As in the previous subsection, (34a) cannot hold exactly but can be aproximated expanding its right hand side in terms of $\varepsilon_{0}$. From the forms of $n^{\mathrm{I}}$ and $T=T^{\mathrm{II}}$ from (22c) and (22d), and using (34b) in the form $3 n^{\mathrm{II}} k_{B} T=3 p$ with $p$ given by $(22 \mathrm{~b})$, we obtain

$$
\begin{aligned}
m c^{2} n^{\mathrm{I}}+3 n^{\mathrm{II}} k_{B} T= & \frac{m c^{2} N_{0}^{\mathrm{I}}}{R_{0}^{3}}\left(\frac{\rho}{\rho_{0}}\right)+\left[3 \rho_{0}-\frac{m c^{2} N_{0}^{\mathrm{I}}}{(\gamma-1) R_{0}^{3}}\right]\left(\frac{\rho}{\rho_{0}}\right)^{\gamma} F^{2} \varepsilon_{0}+\left[\frac{3 \gamma-2}{(\gamma-1)} \rho_{0}+\frac{m c^{2} N_{0}^{\mathrm{I}}}{3(\gamma-1)^{2} R_{0}^{3}}\right]\left(\frac{\rho}{\rho_{0}}\right)^{2 \gamma-1} F^{4} \varepsilon_{0}^{2} \\
& +\left[\frac{(3 \gamma-2)^{2}}{3(\gamma-1)^{2}} \rho_{0}-\frac{m c^{2} N_{0}^{\mathrm{I}}}{27(\gamma-1)^{3} R_{0}^{3}}\right]\left(\frac{\rho}{\rho_{0}}\right)^{3 \gamma-2} F^{6} \varepsilon_{0}^{3}+O\left(\varepsilon_{0}^{4}\right)
\end{aligned}
$$

so that, considering matter dominated conditions and aiming at the best possible approximation, we identify

$$
\rho_{0}=\frac{m c^{2} N_{0}^{\mathrm{I}}}{R_{0}{ }^{3}}, \quad \frac{1}{\gamma-1}=3 \quad \Rightarrow \quad \gamma=\frac{4}{3}
$$

transforming (35) into

$$
m c^{2} n^{\mathrm{I}}+\frac{3}{2} n^{\mathrm{II}} k_{B} T=\rho\left[1+9\left(\frac{\rho}{\rho_{0}}\right)^{2 / 3} F^{4} \varepsilon_{0}^{2}+11\left(\frac{\rho}{\rho_{0}}\right)^{4 / 3} F^{6} \varepsilon_{0}^{3}+O\left(\varepsilon_{0}^{4}\right)\right]
$$

indicating, as in the previous subsection, that a good approximation to (34a) requires the convergence of the power series inside the square brackets, leading to a condition very similar to (28)

$$
\left(\frac{\rho}{\rho_{0}}\right)^{\xi / 3} F^{2 \xi}\left(\varepsilon_{0}\right)^{\xi} \ll 1, \quad \xi=2,3, . .
$$

The form of $\varepsilon_{0}$ follows, as in the previous subsection, by obtaining with the help of (22b), (22d) and (36) the forms of $p$ and $k_{B} T$ expanded in terms of $\varepsilon_{0}$, leading to

$$
\begin{gathered}
p=\frac{m c^{2} N_{0}^{\mathrm{I}} \varepsilon_{0} F^{2}}{R_{0}^{3}}\left(\frac{\rho}{\rho_{0}}\right)^{4 / 3}\left[1+2\left(\frac{\rho}{\rho_{0}}\right)^{1 / 3} F^{2} \varepsilon_{0}+4\left(\frac{\rho}{\rho_{0}}\right)^{2 / 3} F^{2} \varepsilon_{0}^{2}+O\left(\varepsilon_{0}^{3}\right)\right] \\
k_{B} T=\frac{m c^{2} N_{0}^{\mathrm{I}} \varepsilon_{0} F^{2}}{N_{0}^{\mathrm{II}}}\left(\frac{\rho}{\rho_{0}}\right)^{1 / 3}\left[1+5\left(\frac{\rho}{\rho_{0}}\right)^{1 / 3} F^{2} \varepsilon_{0}+16\left(\frac{\rho}{\rho_{0}}\right)^{2 / 3} F^{2} \varepsilon_{0}^{2}+O\left(\varepsilon_{0}^{3}\right)\right]
\end{gathered}
$$

so that it becomes natural to identify

$$
m c^{2} N_{0}^{\mathrm{I}} \varepsilon_{0}=N_{0}^{\mathrm{II}} k_{B} \hat{T}_{0}, \quad \Rightarrow \quad \varepsilon_{0}=\frac{N_{0}^{\mathrm{II}} k_{B} \hat{T}_{0}}{N_{0}^{\mathrm{I}} m c^{2}}
$$

where $\hat{T}_{0}$ is a suitable constant temperature value for the relativistic component. Using (22), (23), (36) and (40), the state variables take the following exact forms

$$
\begin{gathered}
p=\frac{\left(N_{0}^{\mathrm{II}} k_{B} \hat{T}_{0} / R_{0}^{3}\right) F^{2}}{1-2 \varepsilon_{0}\left(\rho / \rho_{0}\right)^{1 / 3} F^{2}}\left(\frac{\rho}{\rho_{0}}\right)^{4 / 3}=\frac{\left(N_{0}^{\mathrm{II}} k_{B} \hat{T}_{0} / R_{0}^{3}\right) F^{2}}{1-2 \varepsilon_{0}\left(R_{0} / R\right) F^{2}}\left(\frac{R_{0}}{R}\right)^{4} \\
n=\frac{\left(N_{0}^{\mathrm{I}}+N_{0}^{\mathrm{II}}\right)}{R_{0}^{3}}\left(\frac{\rho}{\rho_{0}}\right)\left[1-\varepsilon_{0} F^{2}\left(\frac{\rho}{\rho_{0}}\right)^{1 / 3}\right]^{3}=\frac{\left(N_{0}^{\mathrm{I}}+N_{0}^{\mathrm{II}}\right)}{R^{3}}\left[1-\varepsilon_{0} F^{2}\left(\frac{R_{0}}{R}\right)\right]^{3}
\end{gathered}
$$




$$
\begin{gathered}
k_{B} T=\frac{k_{B} \hat{T}_{0} F^{2}}{\left[1-\varepsilon_{0} F^{2}\left(\rho / \rho_{0}\right)^{1 / 3}\right]^{3}\left[1-2 \varepsilon_{0} F^{2}\left(\rho / \rho_{0}\right)^{1 / 3}\right]}\left(\frac{\rho}{\rho_{0}}\right)^{1 / 3}=\frac{k_{B} \hat{T}_{0} F^{2}}{\left[1-\varepsilon_{0} F^{2}\left(R_{0} / R\right)\right]^{3}\left[1-2 \varepsilon_{0} F^{2}\left(R_{0} / R\right)\right]}\left(\frac{R_{0}}{R}\right) \\
\left(R_{, t}\right)^{2}=\frac{8 \pi G m N_{0}^{\mathrm{I}}}{3 R}+\varepsilon_{0} c^{2}\left(\frac{R_{0}}{R}\right)-k c^{2}
\end{gathered}
$$

The fulfilment of (38) with $\varepsilon_{0}$ given by (40) controls how close the state variables aproximate (34a). However in the present case the ratio $N_{0}^{\mathrm{II}} / N_{0}^{\mathrm{I}}$ must be taken into consideration as well. This will be discussed in section VII. Independently of the equations of state presented so far, the evolution range of the models is necessarily restricted by singularities and other geometric features. We examine these features in the following section.

\section{SINGULARITIES, REGULARITY DOMAIN, SYMMETRY CENTERS AND FLRW LIMIT}

Looking at $\rho p$ and $T$ in equations (22), (31) and (41), pending the determination of $R=R(t)$ by integrating (32) and (42), we can identify the following scalar curvature singularities

$$
\begin{gathered}
R=0 \\
1-\frac{3 \gamma-2}{3(\gamma-1)} \varepsilon_{0} F^{2}\left(\frac{R_{0}}{R}\right)^{3(\gamma-1)}=0
\end{gathered}
$$

The singularity (43a) is marked by a hypersurface $t=$ const, all state variables diverge and is similar to a "big-bang" in FLRW spacetimes. The type (43b) has been identified in previous literature as a "finite density" (FD) singularity [12], [13]. It is, in general, a hypersurface marked by a curve in the $(t, r)$ or $(R, r)$ plane, though it is an unphysical singularity because $p, T$ diverge while $\rho$ remains finite

$$
\left[\frac{\rho}{\rho_{0}}\right]_{\mathrm{FD}}=\left[\frac{3(\gamma-1)}{(3 \gamma-2) \varepsilon_{0} F^{2}}\right]^{1 /(\gamma-1)}
$$

for all comoving observers, except along the center worldline $r=0$ where $F(0)=0$ and so $\left[\rho / \rho_{0}\right]_{\mathrm{FD}}$ diverges. Particle number density also remains finite, as can be verified by inserting (44) into (22c).

Another feature worth remarking is the points in spacetime where

$$
1-\frac{\varepsilon_{0}}{3(\gamma-1)} F^{2}\left(\frac{R_{0}}{R}\right)^{3(\gamma-1)}=0
$$

These points mark a spacetime boundary characterized by an asymptoticaly inflationary or (asymptoticaly de Sitter, "ADS") behavior [13]: $p+\rho \rightarrow 0$ and $n \rightarrow 0$. Although $k_{B} T=p / n$ diverges along (45) (because $n$ vanishes for finite $p$ ), this is not realy a curvature singularity since all polynomial curvature scalars are bounded.

Since for the cases we are interested $(\gamma=4 / 3,5 / 3)$, the FD singularity is marked by larger values of $R / R_{0}$ than the ADS boundary, and since this singularity is spacelike [12] [13], we will consider as the regular evolution domain of the solutions all points of spacetime along the fluid worldlines lying to the future of the FD singularity. These domain can be characterized, for every comoving observer, by either one of the following conditions

$$
\frac{R}{R_{0}}>\left[\frac{(3 \gamma-2) \varepsilon_{0} F^{2}}{3(\gamma-1)}\right]^{1 /(3 \gamma-3)}, \quad \frac{\rho}{\rho_{0}}<\left[\frac{3(\gamma-1)}{(3 \gamma-2) \varepsilon_{0} F^{2}}\right]^{1 /(\gamma-1)}
$$

From the material presented in previous sections, it is obvious that the FLRW limit of the Stephani Universes is given by $\varepsilon_{0}=0 \Rightarrow K=0$ and $N=N_{0}$, so that $\dot{u}_{a}=p^{\prime}=s^{\prime}=0$. Since $\varepsilon_{0}=0$ implies $p=T=0$, this limit leads to a dust FLRW universe, and so a sort of asymptotic dust FLRW state can be reached as the fluid evolves in time. Also, for whatever form of $R(t)$ obtained from integrating (32) and (42), the worldlines marked by $r=r_{c}$ where $f\left(r_{c}\right)=0$, with $f$ given by (2) and (10), characterize the symmetry centers. Along these worldlines, the metric function $\sqrt{g_{\theta \theta}}=L f$, the radius of the orbits of the rotation group, vanishes regularly. Also, $\dot{u}_{a}, p^{\prime}$ vanish, as well 
the radial gradients of all other geometric or thermodynamical variables should also vanish, hence spacetime appears isotropic for observers along symmetry centers. Depending on the functions in (2) and (10), the Stephani Universes admit one $\left(r=0\right.$ for $\left.k_{0}=0,-1\right)$ or two $\left(r=0, \pi\right.$ for $\left.k_{0}=1\right)$ symmetry centers.

The singularities (43a-b) and regular boundary (45) have been examined extensively elsewhere, and so we refer the reader to the appropriate references (see [12] to [15]). Considering (46) for the cases $k=0,-1$ of $F$ in (2) and (10), it is evident that these cases do not allow for the existence of a hypersurface $t=$ const in the regularity domain that is also a regular Cauchy surface. In the case $k=1$ we have two symmetry centers marked by $r=0$ and $r=\pi$, and so the full domain of the radial coordinate is $0 \leq r \leq \pi$. Since in this case we have $0 \leq F=\sin (r / 2) \leq 1$, it follows from (46) that a regular Cauchy hypersurface $t=t_{i}$ can be found in the regularity domain, so that a regular evolution takes place in the range $t_{i}<t<t_{M}$, where $t_{M}$ marks the hypersurface of maximal expansion characterized by $\Theta=0$. In this case, the regular hypersurfaces $t=$ const $>t_{i}$ will be diffeomorphic to the invariant spacelike slices of a "closed" FRLW spacetime.

\section{OBSERVATIONAL FEATURES}

As shown in section IV, the fluid sources of the models approximately satisfy the ideal gas equations of state (15),(24) and (34), provided conditions such as (26), (28), (36) and (38) hold. The evolution of the models towards the present time $\left(t \rightarrow t_{0}, R \rightarrow R_{0}\right)$ approaches that of a FLRW dust cosmology, characterizing a homogeneous and isotropic matter dominated universe. It is not surprising that (15), (24) and (34) approach dust sources, the latter being realy a convenient approximation of ideal gases in a low temperature and pressure limits. However, the "nearly dust FLRW" approximation of the models must be examined by verifying how the observational parameters approach as $t \rightarrow t_{0}$ those associated with FLRW cosmologies.

The resemblance of the field equation (9) with the Friedmann equation of a FLRW spacetime, together with the fact that $\rho=\rho(t), \Theta=\Theta(t)$, leads to an invariant definition of observational parameters $H=\Theta / 3=\dot{L} / L=R, t / R$ and $\Omega=8 \pi G /\left(3 c^{2} H^{2}\right)$ that is also very similar to their FLRW equivalents. Evaluating along $R=R_{0}$, we have

$$
\begin{gathered}
H_{0}^{2}=\frac{8 \pi G}{3 c^{2}} \rho_{0}+\left[\frac{\varepsilon_{0}}{\gamma_{1}}-k\right] \frac{c^{2}}{R_{0}^{2}} \\
\Omega_{0} \equiv \frac{8 \pi G \rho_{0}}{3 c^{2} H_{0}^{2}}=\frac{8 \pi G \rho_{0} R_{0}^{2} / 3 c^{4}}{8 \pi G \rho_{0} R_{0}^{2} / 3 c^{4}+\left(\varepsilon_{0} / \gamma_{1}\right)-k} \\
\Omega_{0}-1=\left(k-\frac{\varepsilon_{0}}{\gamma_{1}}\right) \frac{c^{2}}{\left(H_{0} R_{0}\right)^{2}}
\end{gathered}
$$

generalizing the FLRW parameters

$$
\tilde{H}_{0}^{2}=\frac{8 \pi G}{3 c^{2}} \rho_{0}-k \frac{c^{2}}{R_{0}^{2}}, \quad \tilde{\Omega}_{0}=\frac{8 \pi G \rho_{0}}{c^{2} \tilde{H}_{0}^{2}}=\frac{8 \pi G \rho_{0} R_{0}^{2} / 3 c^{4}}{8 \pi G \rho_{0} R_{0}^{2} / 3 c^{4}-k}
$$

where $\gamma_{1}=3(\gamma-1)$ and all quantities with tildes will henceforth be FLRW quantities. Since we are interested in comparing observations in the Stephani Universes and in FLRW dust cosmologies at $R=R_{0}$, it is useful to expand $H_{0}$ and $\Omega_{0}$ in terms of $\tilde{H}_{0}, \tilde{\Omega}_{0}$ up to first order in $\varepsilon_{0}$

$$
H_{0} \approx \tilde{H}_{0}\left[1+\frac{c^{2}}{\gamma_{1}\left(\tilde{H}_{0} R_{0}\right)^{2}} \varepsilon_{0}+O\left(\varepsilon_{0}^{2}\right)\right], \quad \Omega_{0} \approx \tilde{\Omega}_{0}\left[1-\frac{c^{2}}{\gamma_{1}\left(\tilde{H}_{0} R_{0}\right)^{2}} \varepsilon_{0}+O\left(\varepsilon_{0}^{2}\right)\right], \quad \tilde{\Omega}_{0}-1=\frac{k c^{2}}{\left(\tilde{H}_{0} R_{0}\right)^{2}}
$$

showing that, in general $H_{0}>\tilde{H}_{0}$ and $\Omega_{0}<\tilde{\Omega}_{0}$, and that $H_{0}, \Omega_{0}$ are roughly equal to $\tilde{H}_{0}, \tilde{\Omega}_{0}$ plus corrections of the order $\approx \varepsilon_{0} \tilde{\Omega}_{0} \approx \varepsilon_{0}$. Another important observational quantity is the deceleration parameter $\tilde{q}$, defined for FLRW cosmologies as

$$
\tilde{q}_{0} \equiv-\left[\frac{R_{, t t} R}{R_{, t}^{2}}\right]_{0}=\frac{1}{2} \tilde{\Omega}_{0}
$$


We can compute, with the help of $H$ given by (9) for the Stephani Universes, an equivalent quantity $q_{0}$ and expand it in terms of $\tilde{q}_{0}$

$$
\begin{gathered}
q_{0}=-\left[\frac{H_{, t}+H^{2}}{H^{2}}\right]_{0}=\frac{1}{2} \frac{8 \pi G \rho_{0} R_{0}^{2} / 3 c^{4}+\varepsilon_{0}}{8 \pi G \rho_{0} R_{0}^{2} / 3 c^{4}+\varepsilon_{0} / \gamma_{1}-k}=\frac{\Omega_{0}}{2} \frac{2+\varepsilon_{0}}{2-\varepsilon_{0}}-\frac{\varepsilon_{0}}{2-\varepsilon_{0}} \\
q_{0} \approx \tilde{q}_{0}+\frac{1}{2}\left[1-\frac{\tilde{\Omega}_{0}}{\gamma_{1}}\right] \frac{c^{2}}{\left(\tilde{H}_{0} R_{0}\right)^{2}} \varepsilon_{0}+O\left(\varepsilon_{0}^{2}\right)
\end{gathered}
$$

However the definition of $q$, unlike $H, \Omega$, is coordinate dependent. In order to derive the appropriate equivalent of $\tilde{q}_{0}$ for the models based on the Stephani Universes, we need to derive the general form of the relation between red shift and luminosity distance for local observations along $t=t_{0}, r=r_{0}$.

Consider the simplest form of a radial null vector $k^{a}=\left(k^{t}, k^{r}\right)$ for the metric (1) with $U$ and $L$ given by (20)

$$
k^{t}=\frac{c d t}{d v}=\frac{L}{U}=\frac{R}{1-\varepsilon_{0} \gamma_{2} F^{2}\left(R_{0} / R\right)^{3(\gamma-1)}}, \quad k^{r}=\frac{d r}{d v}= \pm 1, \quad u_{a} k^{a}=-L
$$

The effects of inhomogeneity and anisotropy on red shift observations in $t=t_{0}$ of nearby sources $(z \ll 1)$ can be appreciated from the relation between luminosity distance vs red shift given for the radial light rays (51) by [27]

$$
\begin{gathered}
z=\left[u_{a ; b} \hat{k}^{a} \hat{k}^{b}\right]_{0} \Delta \ell_{0}+\frac{1}{2}\left[u_{a ; b c} \hat{k}^{a} \hat{k}^{b} \hat{k}^{c}\right]_{0} \Delta \ell_{0}^{2}+O\left(\Delta \ell_{0}^{3}\right) \\
\hat{k}^{a}=\left(u_{b} k^{b}\right)^{-1} k^{a}, \quad \hat{k}^{t}=-U^{-1}=-u^{t}, \quad \hat{k}^{r}=\mp L, \quad \Delta \ell_{0}=-\left[u_{b} k^{b}\right]_{0} \Delta \lambda=L_{0} \Delta \lambda
\end{gathered}
$$

where $\Delta \lambda$ is the geodesic parameter of an observed gallaxy. For an expanding, accelerating but shear-free 4-velocity, the quantities involved in (52) are

$$
\begin{gathered}
u_{a ; b} \hat{k}^{a} \hat{k}^{b}=\frac{\Theta}{3}-\dot{u}_{a} \hat{k}^{a} \\
u_{a ; b c} \hat{k}^{a} \hat{k}^{b} \hat{k}^{c}=\frac{\Theta, c}{3} \hat{k}^{c}+\frac{2 \Theta^{2}}{9}-\Theta \dot{u}_{a} \hat{k}^{a}-\dot{u}_{b ; c} \hat{k}^{b} \hat{k}^{c}+\dot{u}_{a} \dot{u}_{b} \hat{k}^{b} \hat{k}^{c}
\end{gathered}
$$

Evaluating these expressions for (20), (21), (48b) and (47), expanding up to first order in $\varepsilon_{0}$ and evaluating at $\left(t_{0}, r_{0}\right)$ yields

$$
z \approx \frac{H_{0}}{c}\left[1 \mp \frac{f_{0} c}{2 H_{0} R_{0}} \varepsilon_{0}\right] \Delta \ell_{0}+\frac{H_{0}^{2}}{2 c^{2}}\left\{3+q_{0}+\varepsilon_{0}\left[\mp\left(1+q_{0}\right) F_{0}^{2}+\frac{\left(\gamma_{1}+5\right) f_{0} c}{2 H_{0} R_{0}} \mp \frac{\left(1-2 k F_{0}^{2}\right) c^{2}}{2\left(H_{0} R_{0}\right)^{2}}\right]\right\} \Delta \ell_{0}^{2}+O\left(\Delta \ell_{0}^{3}\right)
$$

where $H_{0}, q_{0}$ are given by (47a) and (50). As shown by (54), the red shift observed from a given source depends in general on the position of the observer (its radial coordinate $r_{0}$ determining $f_{0}, F_{0}$ ). This is expected, since the metric given by (1) and (20) has an explicit radial dependence contained in the functions $f=4 F F^{\prime}$, and so the red shift distribution is anisotropic because of the inhomogeneity of the cosmological model. In particular, an accelerating and shear-free fluid leads to a monopole plus dipole term in (53a), since the direction of the 4-acceleration $(\partial / \partial r)$ is a privileged direction. Hence, for a connecting vector in the radial direction $\Delta \ell_{0}$ has a maximal value, while along other directions it must be multiplied by a factor $\cos \Psi$, where $\Psi$ is the telescopic angle [27]. Observations along the symmetry center $r=0$ detect an isotropic distribution

$$
\left.z\right|_{r=0}=\frac{H_{0}}{c} \Delta \ell_{0}+\frac{H_{0}^{2}}{2 c^{2}}\left[3+q_{0} \mp \varepsilon_{0} \frac{c^{2}}{2\left(H_{0} R_{0}\right)^{2}}\right] \Delta \ell_{0}^{2}+O\left(\Delta \ell_{0}^{3}\right)
$$

Equation (54) provides then the maximal magnitude of the corrections to local red shift isotropy that inhomogeneity of spacetime introduces into to the red shift vs luminosity distance. From $(47 \mathrm{c})$ and since $f_{0} \approx F_{0}$, these corrections are of the order $\varepsilon_{0} \Omega_{0} F_{0} \approx \varepsilon_{0} \bar{\Omega}_{0} F_{0} \approx \varepsilon_{0} F_{0}$. For these corrections to be small, we need $\varepsilon_{0} F_{0} \ll 1$, which for the models considered in sections III and IV, means that either $k=1$ (where $0 \leq r \leq \pi$ and $f, F$ are finite), or if $k=0,-1$, the 
domain $r$ of $f, F$ must be necessarily restricted. However, as discussed in section $\mathrm{V}$, the cases $k=0$, -1 do not allow for regular hypersurfaces $t=$ const nor a regular initial Cauchy surface at $t=t_{0}$. For the case $k=1$, if $\varepsilon_{0} \ll 1$ the corrections due to inhomogeneity are small for all $r_{0}$ compared with the isotropic zero order terms $H_{0}, q_{0}$, which means that local observations are overwhelmingly determined by $H_{0}, q_{0}$, leading to a distribution of red shifts that is almost isotropic but whose observational parameters slightly differ from the FLRW parameters $\tilde{H}_{0}$, $\tilde{q}_{0}$. However, because of (49) and (50), $H_{0}, q_{0}$ differ from $\tilde{H}_{0}, \tilde{q}_{0}$ by corrections of order $\varepsilon_{0}$, and so for sufficiently small $\varepsilon_{0}$, compatible with (28) and (38), this difference should be much smaller than the 10-15 percent or so observational uncertainty [28] in the most accurate possible local measurements of $\tilde{H}_{0}$. This will be discussed in the following section.

\section{DISCUSSION AND CONCLUSSION.}

We have presented in previous sections important general features of the subclass of Stephani Universes derived in sections III and IV. We still need to prove that the approximation to ideal gas equations of state (conditions (28) and (38)), as well as the approximation to isotropic local observations, are actualy met for physicaly reasonable numerical values of the parameters involved. We also need to integrate the Friedmann equations (32) and (42) in order to obtain a fuly determined dynamical evolution of the models, allowing us to verify their prediction of the "age of the universe today" in comparison with the prediction of standard cosmology (dust FLRW universes). Each case, $\gamma=4 / 3$ and $\gamma=5 / 3$, is examined separately below.

\section{A. The classical monatomic ideal gas source.}

For this model the state variables $\rho, p, n, T$ are given by (22a) and (31a-c), satisfying (15) and providing (if (28) holds) a close approximation to (24). Consider matter dominated conditions along $t=t_{0}$, so that $\rho_{0}$ is matter-energy density today. In order to examine the fulfilment of $(28)$, suppose that $m$ is a proton mass and (say) $\hat{T}_{0} \approx 100$ degrees $\mathrm{K}$, leading to $\varepsilon_{0} \approx 10^{-10}$ in $(30)$. The terms of the form (28) in the series in (27) have the form

$$
\left(\frac{\rho}{\rho_{0}}\right)^{2 \xi / 3} F^{\xi} \varepsilon_{0}^{\xi}=F^{\xi}\left(\frac{\rho}{\rho_{0}}\right)^{2 \xi / 3}\left(\frac{k_{B} \hat{T}_{0}}{m c^{2}}\right)^{\xi} \approx\left(10^{-10}\right)^{\xi} F^{\xi}\left(\frac{\rho}{\rho_{0}}\right)^{2 \xi / 3} \ll 1, \quad \xi=2,3, . .
$$

controling the goodness of the approximation of (27) to (24) along the directions of increasing $r$ (increasing $F$ ) and $t$ (decreasing $\rho$ ). For the cases $k=0,-1$, the function $F$ increses monotonously as $r$ increases, so that even for very small values of $\varepsilon_{0}$ and $\rho / \rho_{0}$ we might have a diverging series in (27), since $F^{\xi}<F^{\xi+1}$ for $r>2$. Hence in the cases $k=0,-1$, a necessary (but not sufficient) condition for $(27)$ to converge into $(24)$ is $r<2$, restricting the domain of regularity to comoving observers near the center worldline $r=0$. In the case $k=1$, the function $F$ is bounded and satisfies $F^{\xi}>F^{\xi+1}$ for all values of $\xi$ and $0 \leq r \leq \pi$. Therefore, in this case the convergence of the series in (27) and the fulfilment of (56) only depends on the comparison of $\varepsilon_{0}$ with ratio $\rho / \rho_{0}$ for the leading term of nonzero order $(\xi=2)$ in $(27)$. From (46) and using the value $\varepsilon_{0} \approx 10^{-10}$, the allowed values of $\rho / \rho_{0}$ (with the maximal value at the FD density) is given by

$$
\frac{\rho}{\rho_{0}}<\left[\frac{2}{3 \varepsilon_{0} F^{2}}\right]^{3 / 2} \approx \frac{10^{15}}{\sin ^{3}(r / 2)}
$$

Considering (56) with $\xi=2$ for $k=1$, the conditions for a good approximation of (24) become

$$
\varepsilon_{0}^{2} \sin ^{2}(r / 2)\left(\frac{\rho}{\rho_{0}}\right)^{4 / 3} \approx 10^{-20}\left(\frac{\rho}{\rho_{0}}\right)^{4 / 3} \ll 1, \quad \Rightarrow \quad 10^{-15} \frac{\rho}{\rho_{0}} \ll 1
$$

Thus, comparing (57a) and (57b), we see that the approximation to (24) is a good one as long as one excludes very early evolution stages close to the FD density. However, from $(29 \mathrm{~b})$ and $(31 \mathrm{c})$, a ratio $\rho / \rho_{0} \approx 10^{15}$ can be associated with to $T / \hat{T}_{0} \approx 10^{10}$, corresponding to relativistic temperatures far above the range validity of the classical equation of state (15), (24). Hence, the condition (57b) is perfectly reasonable and allows for a large and physicaly justified range of validity for the approximation of (27) to (24), based on the state variables (31). A convenient limit of this validity range can be set for $\rho / \rho_{0}<10^{6}$, corresponding approximately to $T / \hat{T}_{0}<10^{4}$, roughly (and slightly larger 
than) the range of post-decoupling temperatures for which the evolution a classical ideal gas without interacting with radiation can be justified. The reasoning applied above for the case $k=1$ is valid for $k=0,-1$, as long as we consider only spacetime sections with $r<2$.

The range of validity of the ideal gas equation of state affects also the question, raised in the previous section, on whether local observations on the models are significantly different from those performed in dust FLRW cosmologies. As far as the parameters $H_{0}, \Omega_{0}, q_{0}$ are concerned, the small value $\varepsilon_{0} \approx 10^{-10}$ is sufficient to make them (via (49), (50b)) practicaly identical to the equivalent FLRW parameters. However, the red shift vs luminosity distance (54) contains products of the form $\varepsilon_{0} F_{0}^{2}$ and $\varepsilon_{0} f_{0}^{2}$, and so for the cases $k=0,-1$, these terms can be large even for such a small $\varepsilon_{0}$. Hence, the same reasoning applies: for observations to be close (up to order $\varepsilon_{0}$ ) to FLRW spacetimes, either we assume $k=1$ or, if $k=0,-1$, then we must only consider comoving observers near $r=0$.

The dynamical evolution of the models, especificaly the model $k=1$, follows from the integral of the Friedmann equation (32), leading to

$$
H_{0}\left(t-t_{i}\right)=\frac{1}{\sqrt{\nu_{0}}} \int_{A_{i}}^{A} \frac{\bar{A} d \bar{A}}{\left[\varepsilon_{0} / 2+\mu_{0} \bar{A}-\bar{A}^{2}\right]^{1 / 2}}=\frac{1}{\sqrt{\nu_{0}}}\left[\mu_{0} \arcsin \left(\frac{2 \bar{A}-\mu_{0}}{\sqrt{\mu_{0}^{2}+2 \varepsilon_{0}}}\right)-\left[\varepsilon_{0} / 2+\mu_{0} \bar{A}-\bar{A}^{2}\right]^{1 / 2}\right]_{A_{i}}^{A}
$$

where

$$
A \equiv \frac{R}{R_{0}} \quad \nu_{0}=\frac{c^{2}}{\left(R_{0} H_{0}\right)^{2}}=\frac{2\left(\Omega_{0}-1\right)}{2-\varepsilon_{0}}=\frac{2\left(2 q_{0}-1\right)}{4-\varepsilon_{0}}, \quad \mu_{0}=\frac{8 \pi G}{3 c^{4}} \rho_{0} R_{0}^{2}=\frac{\left(2-\varepsilon_{0}\right) \Omega_{0}}{2\left(\Omega_{0}-1\right)}=\frac{\left(2-\varepsilon_{0}\right) q_{0}+\varepsilon_{0}}{2 q_{0}-1}
$$

and $A_{i}$ is the value of $A$ associated with a suitable initial surface marked by $t=t_{i}$, for example the surface characterized by $T / \hat{T}_{0}=10^{4}$ corresponding (from (31c)) approximately to $R / R_{0} \approx 10^{-2}$. The regularity domain, starting (say) at $A_{i}=R_{i} / R_{0}=10^{-2}$, must exclude unphysical features like the FD singularity and ADS boundary, marked by the coordinate values

$$
\begin{array}{ll}
{\left[\frac{R}{R_{0}}\right]_{\mathrm{FD}}=A_{\mathrm{FD}}=\sqrt{\frac{3 \varepsilon_{0}}{2}} \sin (r / 2) \leq \sqrt{\frac{3 \varepsilon_{0}}{2}}, \quad \text { FD singularity }} \\
{\left[\frac{R}{R_{0}}\right]_{\mathrm{ADS}}=A_{\mathrm{ADS}}=\sqrt{\frac{\varepsilon_{0}}{2}} \sin (r / 2) \leq \sqrt{\frac{\varepsilon_{0}}{2}}, \quad \text { ADS boundary }}
\end{array}
$$

corresponding to very early stages of the evolution $\left(R / R_{0} \approx 10^{-5}\right)$, well outside the stage where the initial value surface $R / R_{0}=10^{-2}$ has been suggested. This surface is a regular Cauchy surface and the evolution range of the models is given by

$$
A_{\mathrm{FD}} \ll A_{i}=10^{-2} \leq A \leq A_{\mathrm{MAX}}=\frac{\mu_{0}}{2}+\sqrt{\left(\frac{\mu_{0}}{2}\right)^{2}+\frac{\varepsilon_{0}}{2}} \approx \mu_{0}
$$

where $A_{\mathrm{MAx}}$ marks the surface of "maximal expansion" characterized by $H=\Theta / 3=0$, after which the fluid recollapses following a time symmetric pattern. From $(58 \mathrm{~b})$ and $(60)$, and bearing in mind that $\varepsilon_{0} \approx 10^{-10}$, we have $A_{\mathrm{MAx}} \approx$ $\Omega_{0} /\left(\Omega_{0}-1\right)$, a very close fit to the value of $A_{\mathrm{MAx}}$ obtained for a FLRW dust universe with $k=1$.

The time required for the fluid to expand up to $A=1$ (the "age of the universe today") and up to $A_{\mathrm{Max}}$ follows directly from (58) and can be expressed as a function of either $\left(\Omega_{0}, \varepsilon_{0}\right)$ or $\left(q_{0}, \varepsilon_{0}\right)$. As shown by figure 1 , the curve relating $H_{0}\left(t_{0}-t_{i}\right)$ obtained from $(58 \mathrm{a}-\mathrm{b})$ vs $\Omega_{0}>1$ for $\varepsilon_{0}=10^{-10}$ is almost identical with the curve relating these same quantities for a FLRW dust universe with $k=1$. As shown in Figure 2 , for any given $\Omega_{0}>1$ the value of $H_{0}\left(t_{0}-t_{i}\right)$ obtained from a dust FLRW universe with $k=1$ is slightly larger than this same quantity obtained from (58). In fact, the ratio of the difference of these values of $H_{0}\left(t_{0}-t_{i}\right)$ to the FLRW value is of the order of $10^{-4}$ of the latter value. Therefore, the age of the universe today predicted by this model is practicaly the same as that predicted by a dust FLRW universe with $k=1$, with $H_{0}\left(t_{0}-t_{i}\right) \rightarrow 2 / 3$ as $\Omega_{0} \rightarrow 1$, thus sharing the same "age problem" associated with standard cosmology. Depending on the value of $H_{0}$, in the range $50-70 \mathrm{~km}(\mathrm{mpc} \mathrm{s})^{-1}$, standard cosmology leads to a range of age values $t_{0}-t_{i} \approx 10^{9-12}$ years that barely meets accepted age values obtained from globular clusters and from nuclear and geological estimates [28]. The same result follows for models with $k=-1$ (assuming $r \ll 1$ ), the "age of the universe" is almost the same as that predicted by standard cosmology with $k=-1$. 
The case $k=0$ (also assuming $r \ll 1$ ) is slightly different, since in the FLRW models $k=0$ necessarily implies $\Omega_{0}=1$, but for the $k=0$ Stephani case the value $k=0$ corresponds to $\Omega_{0}<1$. We can only have $\Omega_{0}=1$ in the Stephani models if $k=1$ and $\varepsilon_{0}=3(\gamma-1)$, a possible but undesirable combination of values since $\varepsilon_{0} \approx 10^{-10}$ while $\gamma=4 / 3,5 / 3$.

From equations (31) for $k=1$ we have $p(t, 0)=T(t, 0)=0$ along the symmetry center $r=0$, while both $p$ and $T$ have their maximal value along any surface $t=$ const along the other symmetry center $r=\pi$ (see subsection $\mathrm{C}$ for discussion on these points), that is, there is a "cold center" along $r=0$ and a "hot center" along $r=\pi$. However, if (28) and (56) hold and for matter dominated conditions, the variation of $p$ and $T$ at any surface $t=$ const is minimal (of the order $\varepsilon_{0} \approx 10^{-10}$ ) as illustrated by the approximated forms of state variables at $t=t_{0}$

$$
\rho_{0}=\frac{m c^{2} N_{0}}{R_{0}^{3}}, \quad p_{0} \approx \frac{N_{0} k_{B} \hat{T}_{0}}{R_{0}^{3}} \sin ^{2}(r / 2)\left[1+O\left(\varepsilon_{0}\right)\right], \quad n_{0} \approx \frac{N_{0}}{R_{0}^{3}}\left[1+O\left(\varepsilon_{0}\right)\right], \quad T_{0} \approx \hat{T}_{0} \sin ^{2}(r / 2)\left[1+O\left(\varepsilon_{0}\right)\right]
$$

clearly indicating a matter dominated regime $p_{0} / \rho_{0} \approx \varepsilon_{0} \ll 1$, with very low pressure and temperature values providing an excelent approximation to dust-like conditions. The models with $\gamma=5 / 3$ might not be realistic becouse they do not allow for the description of another gas (CBMW radiation or other relique gases) as parts of a decoupled mixture accompanying the monatomic ideal gas. However, if the matter content of the present universe is dominated overwhelmingly by non-barionic CDM, and if we can justify this CDM to satisfy the equation of state of a classical monatomic ideal gas ((15) and (24)), then the case $\gamma=5 / 3$ could provide a nice toy model of a CDM universe in its latter stages approaching near dust conditions. Obviuosly, it would be necessary in this case to infere the mass of the particles forming the CDM ideal gas, so that an appropriate value of $\varepsilon_{0}$ be given.

\section{B. The matter-radiation mixture.}

The state variables for the case $\gamma=4 / 3$ follow from (41a-c). This case is perhaps more interesting than the case examined before because of the possibility of accomodating a joint description of non-relativistic matter and a relativistic relic gas. Considering the non-relativistic and ultra-relativistic particles to be barions and photons, the ratio $N_{0}^{\mathrm{II}} / N_{0}^{\mathrm{I}}$ can be associated with the ratio of photons to baryons, and so we have $N_{0}^{\mathrm{II}} / N_{0}^{\mathrm{I}} \approx 10^{8}$ (considering $h \approx .6$ and $\Omega_{B} \approx \Omega_{0}[29]$ ). Assuming $m$ to be a proton mass and $\hat{T}_{0} \approx 3$ degrees K., a good numerical estimate in (40) is $\varepsilon_{0} \approx 3 \times 10^{-5}$, a value five orders of magnitude larger than $\varepsilon_{0}$ in (30). However $\varepsilon_{0}$ is still sufficiently small so that (38) might hold and (37) might provide a good approximation for (34) over a wide temperature and density ranges. Since $T$ under $(34 \mathrm{a}-\mathrm{b})$ is the temperature of the photon gas (so that $\hat{T}_{0} \approx 3$ degrees $\mathrm{K}$ ), we can set again $\rho_{0}$ to be matter-energy density today (overwhelmingly rest mass density).

The arguments discussed in the previous subsection regarding the convergence of the series in (27) for cases $k=0,-1$ apply also for the series in (37). Hence, we consider only the case $k=1$, though the results can be applied to the cases $k=0,-1$ for comoving observers near $r=0$. As before, we use look at (46), now with the value $\varepsilon_{0} \approx 10^{-5}$, obtaining the allowed values of $\rho / \rho_{0}$ (with the maximal value at the FD density) given by

$$
\frac{\rho}{\rho_{0}}<\left[\frac{1}{2 \varepsilon_{0} F^{2}}\right]^{3} \approx \frac{10^{15}}{\sin ^{6}(r / 2)}
$$

Considering (38) with $\xi=2$ for $k=1$, the conditions for a good approximation to (34) become

$$
\varepsilon_{0}^{2} \sin ^{2}(r / 2)\left(\frac{\rho}{\rho_{0}}\right)^{2 / 3} \approx 10^{-10}\left(\frac{\rho}{\rho_{0}}\right)^{2 / 3} \ll 1, \quad \Rightarrow \quad 10^{-15} \frac{\rho}{\rho_{0}} \ll 1
$$

Hence, from (62a) and (62b), a good approximation of (37) to (34a) requires the exclusion of evolution stages close to the FD density. From (39b) and (41c), the ratio $\rho / \rho_{0} \approx 10^{15}$ corresponds to $T / \hat{T}_{0} \approx 10^{5}$, a higher value than temperatures of the matter-radiation decoupling era, thus the range of validity for the approximation of (37) to (34) exceeds that of a decoupled mixture.

The dynamical evolution for the case $k=1$ follows from the following integral of the Friedmann equation (42)

$$
H_{0}\left(t-t_{i}\right)=\sqrt{1-\varepsilon_{0}} \int_{A_{i}}^{A} \frac{\sqrt{\bar{A}} d \bar{A}}{\sqrt{\Omega_{0}-\varepsilon_{0}-\left(\Omega_{0}-1\right) \bar{A}}}=\sqrt{\frac{1-\varepsilon_{0}}{\Omega_{0}-1}}\left[\xi_{0} \arcsin \left(\frac{\bar{A}}{\xi_{0}}-1\right)-\bar{A}^{1 / 2}\left(2 \xi_{0}-\bar{A}\right)^{1 / 2}\right]_{\bar{A}=A_{i}}^{\bar{A}=A}
$$




$$
\text { where: } \quad A \equiv \frac{R}{R_{0}}, \quad \xi_{0} \equiv \frac{\Omega_{0}-\varepsilon_{0}}{2\left(\Omega_{0}-1\right)}
$$

The regularity domain, starting at $\rho / \rho_{0} \approx 10^{15}$ or $R / R_{0} \approx 10^{-5} \approx \varepsilon_{0}$ must exclude the FD singularity and ADS boundary marked by

$$
\begin{aligned}
& {\left[\frac{R}{R_{0}}\right]_{\mathrm{FD}}=A_{\mathrm{FD}}=2 \varepsilon_{0} \sin ^{2}(r / 2) \leq 2 \varepsilon_{0}, \quad \text { FD singularity }} \\
& {\left[\frac{R}{R_{0}}\right]_{\mathrm{ADS}}=A_{\mathrm{ADS}}=\varepsilon_{0} \sin ^{2}(r / 2) \leq \varepsilon_{0}, \quad \text { ADS boundary }}
\end{aligned}
$$

indicating that we can set $A_{i}=A\left(t_{i}\right)$ to a value greater than $2 \varepsilon_{0} \approx 10^{-5}$ that would correspond to the decoupling era $\left(T / \hat{T}_{0} \approx 10^{3}\right)$, for example: $A_{i}=10^{-3}$. The regular evolution range of the model extends from $A_{i}$ up to the value $A_{\mathrm{MAx}}$, marking the maximal expansion $\Theta=0$, where the fluid bounces and begins to collapse in a time symmetric pattern. Evaluating $A_{\mathrm{MAx}}$ from (63) yields

$$
A_{\mathrm{MAX}}=2 \xi_{0}=\frac{\Omega_{0}-\varepsilon_{0}}{\Omega_{0}-1} \approx \frac{\Omega_{0}}{\Omega_{0}-1}
$$

a value almost equal to that obtained for a dust FLRW universe with $k=1$. The "age of the universe today" can be evaluated by setting $A=1$ in (63). Ploting the resulting value $H_{0}\left(t_{0}-t_{i}\right)$ vs $\Omega_{0}>1$ and comparing with the FLRW spacetime leads to curves almost identical to those shown in figure 1. However, the ratio of differences of $H_{0}\left(t_{0}-t_{i}\right)$ to the FLRW value yields an even closer fit to the FLRW expression (see figure 2). Hence, as in the case $\gamma=5 / 3$, this model presents the same "age problem" of standard cosmology mentioned before. If we assume that the non-relativistic component includes cold dark matter (assuming also that it can be described as an ideal gas), we would have a value $N_{0}^{\mathrm{I}}$ larger by two orders of magnitude, modifying (40) to $\varepsilon_{0} \approx 10^{-7}$. However, this alternative value of $\varepsilon_{0}$ does not change the close fit to the dust FLRW universe, in fact, it makes this fit even closer, since the only way to obtain values of $H_{0}\left(t_{0}-t_{i}\right)$ significantly different from the dust FLRW values is to have a larger $\varepsilon_{0} \approx 1$, though the resulting values of $H_{0}\left(t_{0}-t_{i}\right)$ are then significantly smaller than those of the FLRW model. As in the case $\gamma=5 / 3$, this hopeless situation also occurs for the cases with $k=0,-1$.

As in the previous case, from (39) and (41), we also have a "cold" symmetry center $r=0$ where $p(t, 0)=T(t, 0)=0$ (see subsection $\mathrm{C}$ for discussion on this aspect), and a "hot" center at $r=\pi$ where both $p$ and $T$ have their maximal value along any surface $t=$ const. At $t=t_{0}$ the state variables take the approximated forms

$$
\rho_{0}=\frac{m c^{2} N_{0}^{\mathrm{I}}}{R_{0}^{3}}, \quad p_{0} \approx \frac{N_{0}^{\mathrm{II}} k_{B} \hat{T}_{0}}{R_{0}^{3}} \sin ^{2}(r / 2)\left[1+O\left(\varepsilon_{0}\right)\right], \quad n_{0} \approx \frac{\left(N_{0}^{\mathrm{I}}+N_{0}^{\mathrm{II}}\right)}{R_{0}^{3}}\left[1+O\left(\varepsilon_{0}\right)\right], \quad T_{0} \approx \hat{T}_{0} \sin ^{2}(r / 2)\left[1+O\left(\varepsilon_{0}\right)\right]
$$

so that matter dominated dust-like conditions do prevail at $t=t_{0}$, with the variation of $p$ and $T$ of the order $\varepsilon_{0} \approx 10^{-5}$. Also, as with the case $\gamma=5 / 3$, as long as $F_{0}$ and $f_{0}$ in (54) are bonded, observation parameters are almost identical to their FLRW equivalents. However, the pressure and temperature in this case correspond to the pressure and temperature of the MWCB (with $\hat{T}_{0}=3$ degrees K.). Hence, the ratio $p_{0} / \rho_{0} \approx \varepsilon_{0} \approx 10^{-5}$ from (66), is roughly the accepted value of ratio of the energy density of the CMBR $\left(\approx 3 p_{0}\right)$ to that of visible non-relativistic matter. Regarding the temperature, a deviation of order $\varepsilon_{0} \approx 10^{-5}$ gives an excellent fit with the observed deviations of the isotropy of the temperature of the MWCB [28], 29]. We might consider including CDM in the matter content of the non-relativistic component so that $\varepsilon_{0} \approx 10^{-7}$. This might sound reasonable, but CDM is most possibly non-barionic, and so, as in the case $\gamma=5 / 3$, we would have to justify that it satisfies an equation of state like (34). With or without CDM, the case $\gamma=4 / 3$ could be a nice toy model of a matter dominated universe approaching near dust conditions, though it would be a better model than that provided by $\gamma=5 / 3$ as it would include the MWCB.

\section{The drawbacks.}

It emerges from the discussion in the previous subsections that the $k=1$ models, whether $\gamma=4 / 3$ or $\gamma=5 / 3$, share two unappealing features: (A) The maximal and minimal values of the rest mass density $\left(m c^{2} n\right.$ or $\left.m c^{2} n^{\mathrm{I}}\right)$ respectively occur along the symmetry centers $r=0$ and $r=\pi$, while the maximal and minimal values of the internal 
energy density $\left(\rho-m c^{2} n \approx 3 p / 2\right.$ or $\left.\rho-m c^{2} n^{\mathrm{I}} \approx 3 p\right)$ occur along $r=\pi$ and $r=0$. This means that along $r=0$ all energy density is rest mass energy density and there is a higher density of particles but zero temperature and pressure for all times (see discussion further ahead), while at $r=\pi$ there are less particles by volume but a higher temperature and pressure. (B) The fact that pressure and temperature vanish along $r=0$ for all evolution times (forced by the specific spacial dependence of the metric contained in the function $F$ ) is a very different situation from $p, T$ tending to zero asymptoticaly as the fluid expands and cools. This situation leads to the entropy per particle (18c) diverging as $r \rightarrow 0$, so that the left hand side of the Gibbs equation (14) (Ts'), remains finite (as the right hand side does). Although this feature is problematic, it is consistent with the fact that an absolute zero temperature (if $T$ is stricly zero instead of $T \rightarrow 0$ asymptoticaly) is impossible to attain in equilibrium classical thermodynamics [24]. This feature (appearing also for the cases $k=0,-1$ ) is perhaps the strongest drawback on the physical plausibility of the models.

\section{Final remarks.}

The models derived and presented in this paper are mathematicaly simple, theoreticaly consistent and satisfy physicaly motivated equations of state. Whether one considers it a virtue or a defect, the models bear an extremely close resemblance (observationaly and dynamicaly) to FLRW cosmologies in the matter dominated regime with a dust source, therefore they share the possible failure of matter dominated standard cosmology in dealing with recent observational evidence [28]. However, even if hard core cosmologists might dismiss them as not sufficiently realistic, the solutions can at least be applied as self-consistent cosmological toy models or as useful educational tools in teaching cosmology. Hopefuly the results of the present paper, besides rescueing the Stephani Universes from oblivion, will motivate and improve further research based on this nice and elegant class of solutions.

\section{ACKNOWLEDGMENTS}

This work is supported by grant DGAPA-IN122498. The wise meowing and feline wisdom of Mimi, Tontina, Niña, Chata, Pintita, Martin, Feucho, Bonito, Ulises, Vitorio and Pocholo provided the author with enough inspiration as to make the writing of this paper an enjoyable venture.

[1] D. Kramer, H. Stephani, M.A.H. MacCallum, E. Herlt,Exact solutions of Einstein's field equations,(Cambridge University Press, Cambridge, U.K, 1980).

[2] A. Krasinski, Inhomogeneous Cosmological Models, (Cambridge University Press, Cambridge, U.K, 1998).

[3] W.B. Bonnor and M.C. Faulkes, MNRAS, 137, 239, (1967).

[4] I.H. Thomson and G.J. Whitrow, MNRAS, 136, 207,(1967).

[5] I.H. Thomson and G.J. Whitrow, MNRAS, 139, 499,(1969).

[6] H. Nariai and K. Tomita K, Progr. Theor. Phys.,40, 679, (1968).

[7] H. Bondi, MNRAS (Physics), 142, 333, 1969

[8] R.N. Henriksen, A.G. Emslie and P.S. Wesson, Phys. Rev. D, 27, 1219, (1983).

[9] J. Ponce de Leon and P.S. Wesson, Phys. Rev. D, 39, 420, (1989).

[10] A. Cook, Austr. J. Phys.,28, 413, 1975

[11] A. Krasinski, Gen. Rel. Gravit., 15, 673, 1983.

[12] R.A. Sussman, Jou. Math. Phys.,29, 945, (1988).

[13] R.A. Sussman, Jou. Math. Phys.,29, 1177, (1988).

[14] R.A. Sussman, Phys. Rev. D, 40, 1364, (1989).

[15] M. Dabrowski, Jou. Math. Phys.,34, 1447, (1993).

[16] C. Bona and B. Coll, Gen. Rel. Gravit.,20, 297, (1988).

[17] H. Quevedo and R.A. Sussman, Jou. Math. Phys., 36, 1365, (1995).

[18] A. Krasinski, H. Quevedo and R.A. Sussman, Jou. Math. Phys., 38, 2602, (1997).

[19] M. Dabrowski, Ap. Jou., 447, 43, (1995).

[20] M. Dabrowski and M. Hendry, Ap. Jou., 498, 67, (1998).

[21] R. Mansouri, Ann. Inst. H. Poincare, 27, 175, (1977) 
[22] B. Mashhoon and H. Partovi, Ann. Phys. NY, 4, 137, (1982).

[23] S.R de Groot, W.A. van Leeuwen and Ch.G. van Weert, Relativistic Kinetic Theory, (North Holland Publishing Co., 1980).

[24] K. Huang, Statistical Mechanics, (J Wiley and sons, 1978).

[25] R.A. Sussman and J.Triginer, Class. and Quantum Grav., 15, 1769, (1999).

[26] R.A. Sussman and D. Pavon, LANL pre-print archive number gr-qc/9907010. To appear in Phys. Rev. D, (1999).

[27] G.F.R. Ellis and M.A.H. MacCallum, Comm. Math. Phys.,19, 31, (1970).

[28] J. Peacock, Cosmological Physics, (Cambridge University Press, Cambridge, U.K, 1999).

[29] E.W. Kolb and M.S. Turner, The Early Universe, (Adison Wesley Publishing Co.,1990).

FIG. 1. Age of universe today. This figure displays $H_{0}\left(t_{0}-t_{i}\right)$ vs $\Omega_{0}$ for the $k=1$ model with $\gamma=5 / 3$ (solid line) and for a $k=1$ FLRW cosmology with a dust source. The values of $H_{0}\left(t_{0}-t_{i}\right)$ follow from (58) with $A=1$ and $A_{i}=10^{-3}$, with $H_{0}=60 \mathrm{~km}(\mathrm{mpc} \mathrm{seg})^{-1}$. Notice the almost identical fit between both cosmological models. The same close fit (not displayed) with standard cosmology occurs for $k=-1$, as well as for the $k=1, k=-1$ models with $\gamma=4 / 3$. For the case $k=0$ standard cosmolgy yields $\Omega_{0}=1$, while for the Stephani models (either $\gamma=4 / 3,5 / 3$ ) we would obtain a plot analogous to the case $k=1$ with $\Omega_{0}>1$. These (and the remaining) plots were obtained with the help of the symbolic computing package Maple V.

FIG. 2. The relative ratio of the differences of $H_{0}\left(t_{0}-t_{i}\right)$ for the Stephani and FLRW models. This plot displays the ratio $X_{0} \equiv \log _{10}\left|\tilde{Y}_{0}-Y_{0}\right| / \tilde{Y}_{0}$ as a function of $\Omega_{0}$ for the case $k=1$ of $\gamma=4 / 3,5 / 3$, where $Y_{0} \equiv H_{0}\left(t_{0}-t_{i}\right)$ and $\tilde{Y}_{0}$ is the FLRW equivalent. The smallness of this ratio illustrates the close fit in the dynamics of the Stephani models and standard cosmology. The resemblance is tighter for $\gamma=4 / 3$ (curve below) than for the model $\gamma=5 / 3$ (curve above). 


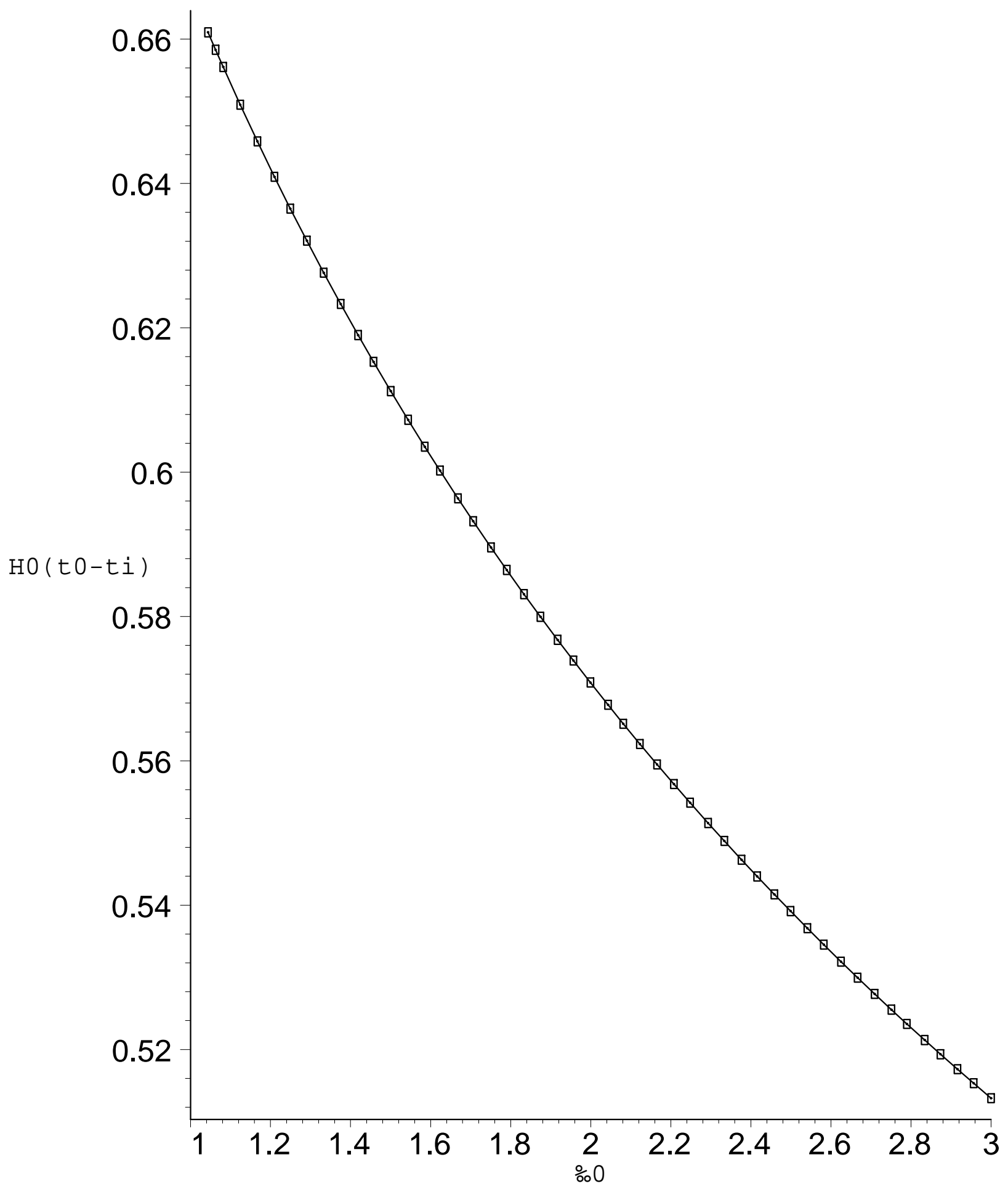




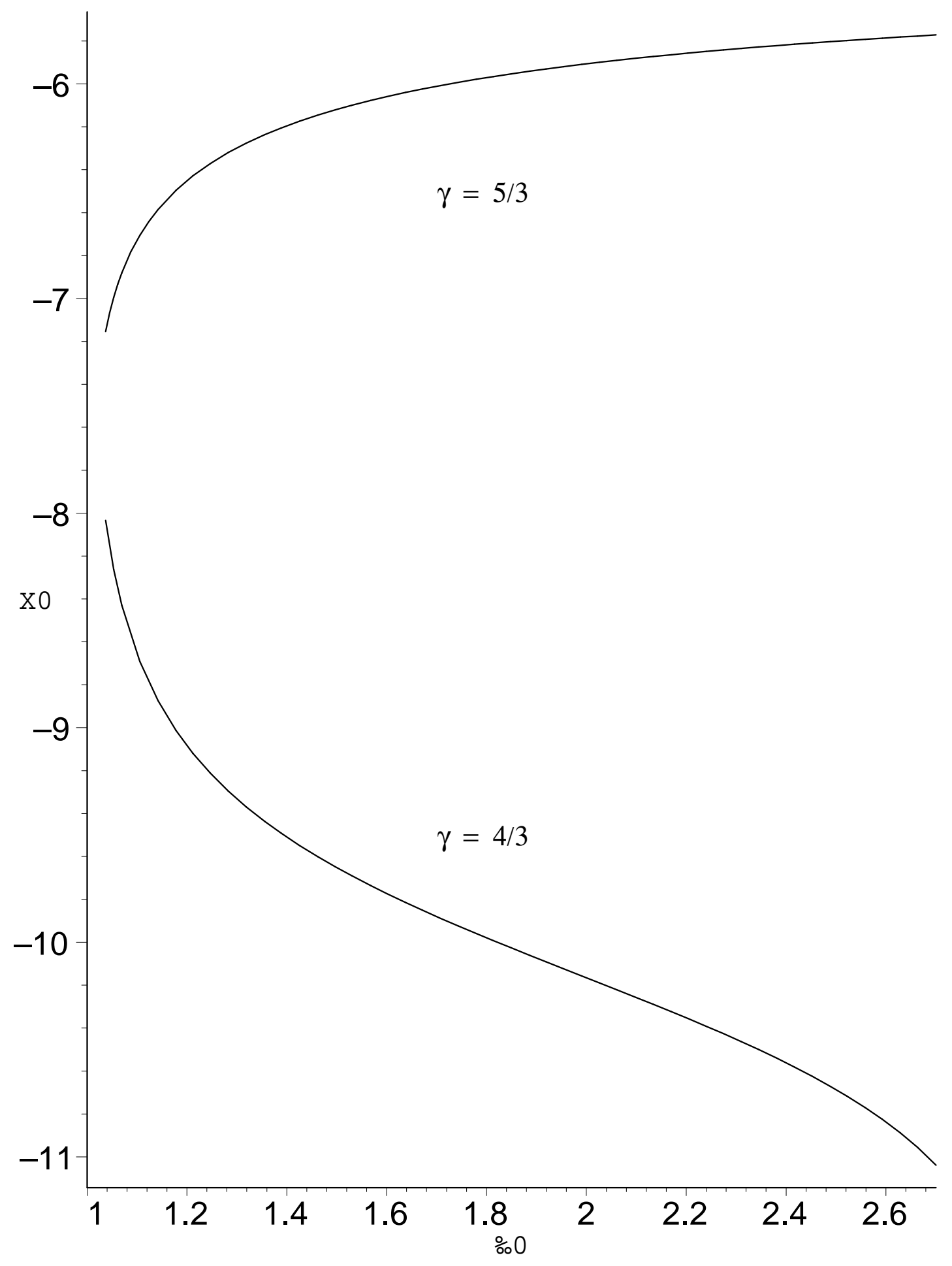

